\title{
Diverse forms of bonding in two-dimensional Si allotropes: Nematic orbitals in the $\mathrm{MoS}_{2}$ structure
}

\author{
Florian Gimbert, ${ }^{1}$ Chi-Cheng Lee, ${ }^{1}$ Rainer Friedlein, ${ }^{1}$ Antoine Fleurence, ${ }^{1}$ Yukiko Yamada-Takamura, ${ }^{1}$ and Taisuke Ozaki ${ }^{1,2}$ \\ ${ }^{1}$ School of Materials Science, Japan Advanced Institute of Science and Technology, 1-1 Asahidai, Nomi, Ishikawa 923-1292, Japan \\ ${ }^{2}$ Research Center for Simulation Science, Japan Advanced Institute of Science and Technology, 1-1 Asahidai, Nomi, Ishikawa 923-1292, Japan
}

(Received 17 January 2014; revised manuscript received 11 June 2014; published 17 October 2014)

\begin{abstract}
The interplay of $s p^{2}$ - and $s p^{3}$-type bonding defines silicon allotropes in two- and three-dimensional forms. A two-dimensional phase bearing structural resemblance to a single $\mathrm{MoS}_{2}$ layer is found to possess a lower total energy than low-buckled silicene and to be stable in terms of its phonon dispersion relations. A set of cigar-shaped nematic orbitals originating from the $\mathrm{Si} s p^{2}$ orbitals realizes bonding with a sixfold coordination of the inner $\mathrm{Si}$ atoms of the layer. The identification of these nematic orbitals advocates diverse Si bonding configurations different from those of $\mathrm{C}$ atoms.
\end{abstract}

DOI: 10.1103/PhysRevB.90.165423

PACS number(s): 73.22.-f, 61.46.-w, 81.05.Zx

\section{INTRODUCTION}

With graphene at the forefront of attention, with its unique and exotic properties, at present two-dimensional materials are experiencing an explosion of interest in scientific and technological aspects [1]. While the excellent electronic properties of graphene are derived from its structural robustness, the same property makes it a challenging task to engineer the optical and transport properties. This challenge is stimulating the search for alternative two-dimensional layered materials that are more flexible in terms of their structural and electronic properties [2,3]. In this context, in particular, two new promising two-dimensional materials with a honeycomb structure made of silicon or germanium atoms have been studied as theoretical objects since 1994 [4]. Most importantly, in their still hypothetical, slightly buckled, free-standing forms, silicene and germanene, as they have come to be called [5], exhibit a band structure similar to that of graphene, merging linear dispersions of $\pi$ and $\pi^{*}$ bands at the Fermi level to form Dirac cones at the $K$ points $[2,4,5]$. Experimentally, it has been shown that two-dimensional Si honeycomb lattices can be formed epitaxially on Er layers [6] as well as on the $\mathrm{Ag}(111)$ [7-9], $\mathrm{ZrB}_{2}$ (0001) [3], and $\operatorname{Ir}(111)$ [10] surfaces. It is established that the interactions with the substrates have a distinct influence on the structural and electronic properties of the layers [11-13]. No experimental evidence for the existence of germanene has been reported yet.

As density functional theory (DFT) calculations have so far consistently predicted that low-buckled (LB) silicene is the most stable form of free-standing Si allotropes, very recently, it has been shown that the addition of $\mathrm{Si}$ adatoms to pristine silicene results in the formation of a dumbbell structure with a lower total energy per atom $[14,15]$. Interestingly, an even higher cohesive energy can be achieved toward the complete coverage of adatoms. In fact, the periodic dumbbells can be recognized to form the structure of a well-known single layer of $\mathrm{MoS}_{2}$. In this structure, the central $\mathrm{Si}$ atoms are sixfold

Published by the American Physical Society under the terms of the Creative Commons Attribution 3.0 License. Further distribution of this work must maintain attribution to the author(s) and the published article's title, journal citation, and DOI. coordinated. While hypervalent $\mathrm{Si}$ atoms are known to exist in molecular heterocompounds [16], the $s p^{3}$ hybridization realized in the most stable bonding between $\mathrm{Si}$ atoms provides a fourfold coordination. It then needs an explanation of why a sixfold coordination may be formed in a structure made purely by $\mathrm{Si}$ atoms. Therefore, it is timely and interesting to investigate the properties of this new $\mathrm{Si}$ phase beyond the perspective related to the introduction of defects or adatoms to low-buckled silicene.

In this article are reported the results of first-principles calculations in which we investigated the stability of this new Si phase $\left(\mathrm{MoS}_{2}-\mathrm{Si}\right)$ together with a possible similar $\mathrm{Ge}$ allotrope $\left(\mathrm{MoS}_{2}-\mathrm{Ge}\right)$ whose structures are that of a single layer of molybdenum disulfide, or $\mathrm{MoS}_{2}$ [17]. The results are compiled for a wide range of lattice constants and compared to those of other two-dimensional silicon structures. The phonon dispersion further demonstrates that $\mathrm{MoS}_{2}-\mathrm{Si}$ is stable on the Born-Oppenheimer surface. A new form of $\sigma$ bonding expressed by three cigar-shaped orbitals coexists with an extended $\pi$ electronic band structure. The direction of these orbitals has changed from the typical in-plane direction of the orbitals in the $s p^{2}$ hybridization to the out-of-plane direction to form cigar-shaped orbitals. In analogy to the nematic electronic structure [18], the aligned and cigar-shaped orbitals may be called nematic.

\section{COMPUTATIONAL DETAILS}

The DFT calculations within a generalized gradient approximation [19] have been performed using the OPENMX code [20,21], which is based on norm-conserving pseudopotentials generated with multireference energies and optimized pseudoatomic basis functions. The cutoff radius of 7 bohrs has been chosen for all the basis functions. For $\mathrm{Si}$ and $\mathrm{Ge}$ atoms, $s 2 p 2 d 1$ configurations have been adopted. The spin-orbit coupling has not been considered in our calculation. In order to avoid interactions, the distance between supercells is larger than $10 \AA$ in the $z$ direction (the direction perpendicular to the plane of layers). The $k$ mesh has been set to $12 \times 12 \times 1$ for the primitive cell. The structures were fully optimized until the maximum force became less than $10^{-4}$ hartree/bohr. The $k$ mesh and the configurations of basis orbitals have been tested against numerical convergence. In order to calculate the phonon frequencies, a dynamical matrix has been constructed 
by calculating real-space force constants. Using a $12 \times 12$ supercell, the atoms have been chosen to be displaced by $0.02 \AA$ out of the equilibrium positions [22]. In order to plot the symmetry-respecting Wannier functions of $\mathrm{MoS}_{2}-\mathrm{Si}$, the commonly adopted procedure for maximizing the localization of Wannier functions has not been performed [23]. All the geometric structures are plotted using the XCrySDen software. The energy curves for the phases shown in Fig. 2 have also been confirmed by calculations with the first-principles code WIEN2K [24].

\section{RESULTS}

While as a common feature in the two-dimensional $\mathrm{Si}$ allotropes the three $\sigma$ bands show dispersions similar to those of bands in low-buckled silicene, values of bond lengths and buckling heights vary significantly. Our finding suggests that the $\sigma$ bonds between $\mathrm{Si}$ atoms are more flexible than one could expect such that diverse forms of $\sigma$ bonding can allow the existence of a number of $\mathrm{Si}$ allotropes.

As shown in Fig. 1, a single layer of $\mathrm{MoS}_{2}-\mathrm{Si}$ crystallizes in an A-B-A stacking configuration. In preserving the honeycomb structure, the A-B layer taken by itself is exactly the one of free-standing low-buckled silicene. In the $\mathrm{MoS}_{2}$-Si structure, the primitive unit cell contains three atoms in comparison with two atoms in silicene. The middle atom is bound to six atoms while the top and bottom atoms have three neighbors. Clearly, the coordination is very different from that in graphenelike lattices, where each atom is bound to three neighboring atoms.

Next, we compare the total energy per atom and structural parameters of the phases under consideration as a function of the in-plane lattice constant $a$. Since the highly buckled forms of silicene and germanene are unstable [2], we restrict the investigations to lattice parameters in the region of the LB phase. In Fig. 2(a) is shown the relationship between the total

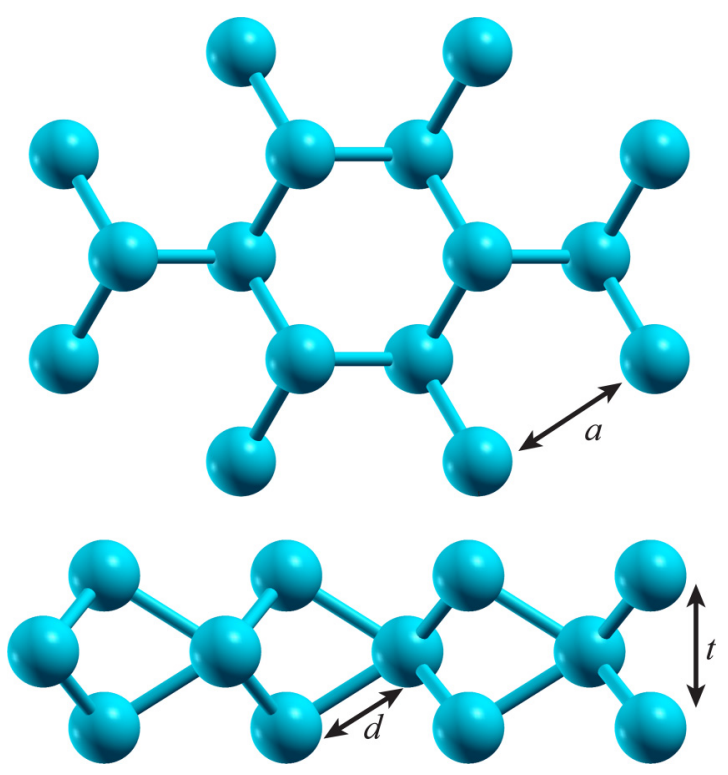

FIG. 1. (Color online) Top and side view of the $\mathrm{MoS}_{2}$-type single layer of $\mathrm{Si}$ atoms with the lattice constant $(a)$, the bonding distance $(d)$, and the thickness $(t)$ indicated.
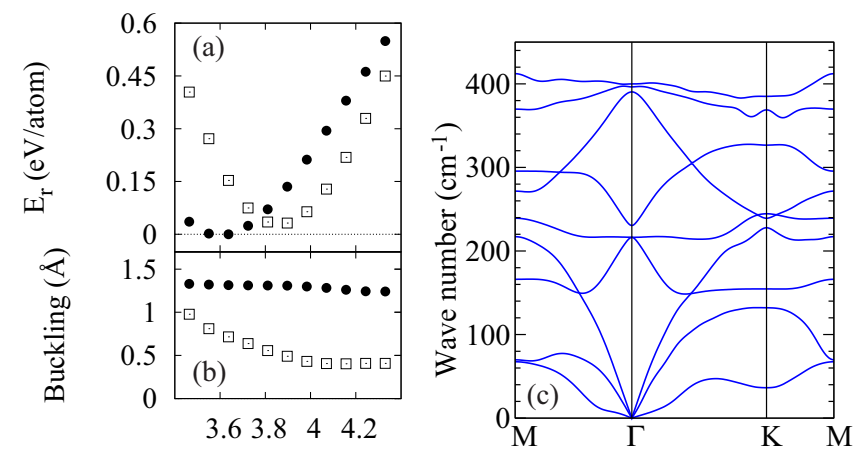

Lattice Parameter $(\AA)$

FIG. 2. (Color online) (a) Relative energy (in $\mathrm{eV} /$ atom) of silicene (empty squares) and $\mathrm{MoS}_{2}-\mathrm{Si}$ (filled circles). (b) Buckling of silicene (empty squares) and $\mathrm{MoS}_{2}-\mathrm{Si}$ (filled circles). For $\mathrm{MoS}_{2}-\mathrm{Si}$, the buckling is defined as the distance between two planes, corresponding to half of the thickness. (c) Phonon dispersion relations of $\mathrm{MoS}_{2}-\mathrm{Si}$ as obtained by the force-constant method.

energy per atom $\left(E_{r}\right)$ and the lattice constant $(a)$, for both LB silicene (empty squares) and $\mathrm{MoS}_{2}-\mathrm{Si}$ (filled circles). The evolution of the buckling is plotted in Fig. 2(b). In order to facilitate comparison between the two phases, for the $\mathrm{MoS}_{2}$ structure, half of the thickness is taken as the value of the buckling. With the energy minimum occurring at a lattice constant of $3.90 \AA$, LB silicene prefers a buckling of $0.49 \AA$ at the energy minimum at $a=3.90 \AA$. These parameters compare well with values reported previously $[2,25,26]$.

Interestingly, in equilibrium, the total energy of $\mathrm{MoS}_{2}-\mathrm{Si}$ is lower than that of LB silicene, stabilized at a shorter lattice constant of $a=3.64 \AA$. Due to the stacking of three atoms, with $2.63 \AA$, the thickness $(t)$ of the $\mathrm{MoS}_{2}$-Si layer is larger than that of silicene. Similar to the Si phases, $\mathrm{MoS}_{2}-\mathrm{Ge}$ possesses a lower total energy than LB germanene as well. The total energy and lattice parameters of both $\mathrm{MoS}_{2}-\mathrm{Si}$ and $\mathrm{MoS}_{2}-\mathrm{Ge}$ are given in Table I.

With $\mathrm{MoS}_{2}$-Si being more stable than LB silicene, it is relevant to understand its stability by investigating the BornOppenheimer surface. This can be done by calculating the phonon frequencies in the harmonic approximation.

The phonon dispersion relations of $\mathrm{MoS}_{2}-\mathrm{Si}$ are shown in Fig. 2(c). The frequencies are overall lower than those of LB silicene, which can be understood from the elongated bond lengths that represent a weaker bonding. No branch with imaginary frequencies is found. This suggests that freestanding $\mathrm{MoS}_{2}-\mathrm{Si}$ is a stable phase that is preferentially formed

TABLE I. Values of the lattice constant ( $a$ in $\AA$ ), the thickness ( $t$ in $\AA$ ), the bonding distance ( $d$ in $\AA$ ), and the relative energy ( $E_{r}$ in $\mathrm{eV} /$ atom) for silicene and $\mathrm{MoS}_{2}$-Si (upper part), as well as germanene and $\mathrm{MoS}_{2}$-Ge (lower part).

\begin{tabular}{lcccc}
\hline \hline & $a$ & $t$ & $d$ & $E_{r}$ \\
\hline Silicene & 3.90 & 0.49 & 2.30 & 0.032 \\
MoS $_{2}$ Si & 3.64 & 2.63 & 2.47 & 0 \\
Germanene & 4.07 & 0.74 & 2.47 & 0.140 \\
MoS $_{2}-\mathrm{Ge}$ & 3.90 & 2.88 & 2.67 & 0 \\
\hline \hline
\end{tabular}



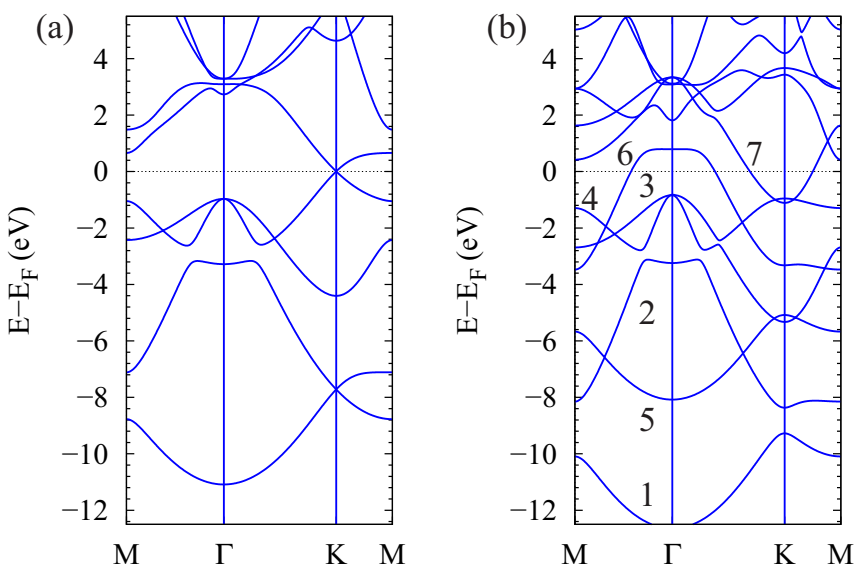

(c)
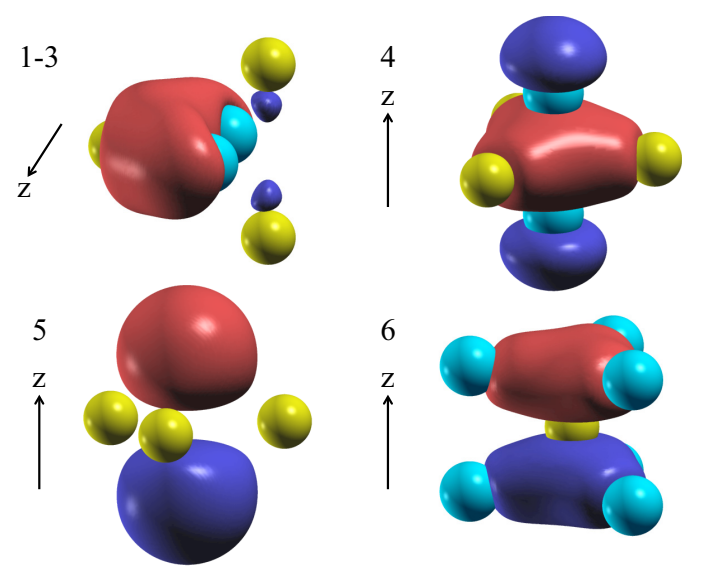

FIG. 3. (Color online) (a) Band structure of low-buckled silicene with a lattice parameter of $a=3.90 \AA$. (b) Band structure of $\mathrm{MoS}_{2}-\mathrm{Si}$ with $a=3.64 \AA$. (c) Symmetry-respecting Wannier functions of $\mathrm{MoS}_{2}-\mathrm{Si}$ related to the bands labeled 1-6 in (b). The top, bottom, and middle $\mathrm{Si}$ atoms are shown with different colors.

instead of the commonly studied low-buckled silicene. For $\mathrm{MoS}_{2}-\mathrm{Ge}$, on the other hand, a small amount of computed imaginary phonon frequencies may indicate a possible structure instability. The following discussion will therefore focus on $\mathrm{MoS}_{2}-\mathrm{Si}$.

The electronic band structures of $\mathrm{LB}$ silicene and $\mathrm{MoS}_{2}-\mathrm{Si}$ are presented in Figs. 3(a) and 3(b), respectively. Given that the bond length and the degree of buckling are larger and that the lattice constants are shorter for $\mathrm{MoS}_{2}-\mathrm{Si}$ as compared to silicene, it is surprising that the band structure is not far away from that of silicene. Major differences observed around the Fermi energy relate to the disappearance of the Dirac cone at the $K$ point typical for the LB silicene and to the appearance of two new bands, labeled 5 and 6 in Fig. 3(b).

To further understand similarities and differences between these two phases, we construct the symmetry-respecting Wannier functions of $\mathrm{MoS}_{2}$-Si [27]. The energy window is chosen to allow for a reproduction of the six occupied bands, labeled 1-6 in Fig. 3(b). As shown in Fig. 3(c), the respective orbitals represented by Wannier functions have contributions in different bands and adopt particular shapes. The orbitals dominating bands 1-3 originate from the $s p^{2}$ orbitals of the middle $\mathrm{Si}$ atom. Interestingly, in order to accommodate bonding between the top and bottom $\mathrm{Si}$ atoms, these orbitals have a nematic shape. For clarity, only one of the three symmetric nematic orbitals is shown in Fig. 3(c). The band dispersions related to the nematic orbitals resemble those of the $\sigma$ bands of LB silicene in Fig. 3(a). Without these nematic orbitals, it is difficult to provide fully occupied $\sigma$ band dispersions that are similar to the ones in LB silicene. Note that for the silicene structure of the A-B stacking obtained directly from the equilibrium lattice parameters of $\mathrm{MoS}_{2}-\mathrm{Si}$ the $\sigma$ bands of silicene can only be partially occupied.

Another interesting finding relates to orbitals with $p_{z}$ contributions: in particular, the fourth orbital is derived from the $p_{z}$ orbitals of the top and bottom $\mathrm{Si}$ atoms and the $s p^{2}$ orbitals of the middle $\mathrm{Si}$ atoms. At the $K$ point, the corresponding band crosses the seventh band, which, however, does not have any $p_{z}$ character. The crossing can therefore not be considered to be derived from an original Dirac point of silicene. The fifth and sixth orbitals form orbitals with $p_{z}$ symmetry having a node at the height of the middle Si atom. As can be recognized in Fig. 3(c), while the fifth orbital is mainly derived from the $p_{z}$ and $s$ orbitals of the top and bottom $\mathrm{Si}$ atoms, the sixth orbital stems from the $s p^{2}$ orbitals of the top and bottom $\mathrm{Si}$ atoms therefore displaying $p_{z}$ symmetry. Although the band dispersions related to the $p_{z}$ orbitals bear some resemblance to those of the $\pi$ and $\pi^{*}$ bands of LB silicene, no Dirac cones are formed since the orbital nature of the two $p_{z}$ orbitals is essentially different.

The modification of the electronic properties as compared to silicene is also evident from the plot of the charge density shown in Fig. 4(a). The top and bottom atoms are bound to the central atom via three of these nematic orbitals, which allow for the coordination of the central atom with its six neighbors. For comparison, in Figs. 4(b) and 4(c) are displayed (a)

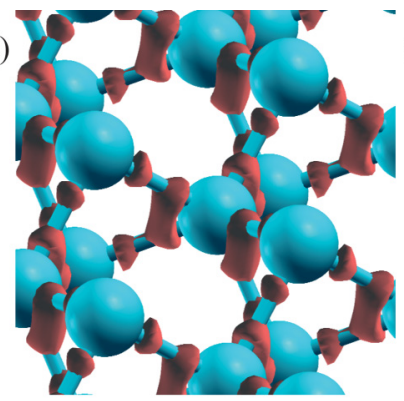

(c)

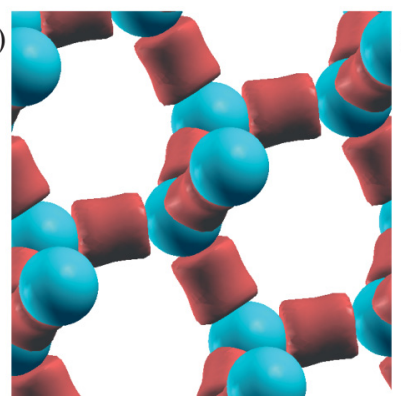

(b)

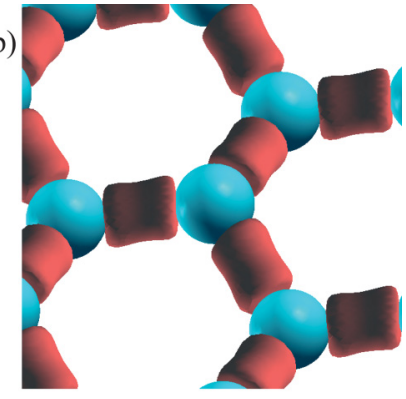

(d)

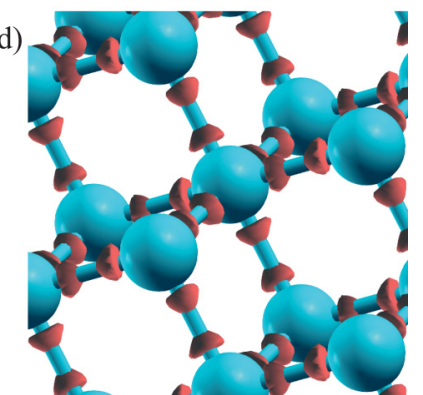

FIG. 4. (Color online) Perspective view (viewing direction close to normal) of valence charge density for different structures composed of Si atoms. (a) $\mathrm{MoS}_{2}-\mathrm{Si}$ layer, corresponding to A-B-A stacking. (b) Low-buckled silicene. (c) Si layer with a diamond structure. (d) A-B-C stacking structure for silicon. 
the charge densities of silicene and of three $\mathrm{Si}(111)$ monolayers in the diamond bulk, in which atoms are three- or fourfold coordinated, respectively. These two structures have a higher total energy per atom than $\mathrm{MoS}_{2}-\mathrm{Si}$. This suggests that for two-dimensional silicon $\sigma$ bonding of the nematic type is preferred. Note that $\sigma$ orbitals in the classical $s p^{2}$ hybridization and nematic orbitals observe the same threefold rotational symmetry.

In order to allow for an even higher flexibility in the bonding, one can imagine twisting the nematic orbitals to form a new A-B-C stacking structure. Such an asymmetric bonding with respect to the planar $s p^{2}$ orbitals is by $180 \mathrm{meV}$ per $\mathrm{Si}$ atom energetically unfavorable. In addition, the middle $\mathrm{Si}$ atom of the A-B-C stacking structure shares a bonding similar to that of the sixfold coordinated Si atom in the $\beta$-tin Si phase that can only be stabilized under a high pressure [28]. The charge density of the A-B-C phase is shown in Fig. 4(d).

\section{CONCLUSION}

To summarize, a two-dimensional Si phase structurally equal to a single $\mathrm{MoS}_{2}$ layer is identified. Within DFT, this phase is stable on the Born-Oppenheimer surface in terms of the total energy and the phonon frequencies. Instead of the commonly accepted bonding configurations in silicene or the diamond structure of silicon that are related to a mixture of $s p^{2}$ and $s p^{3}$ or pure $s p^{3}$ hybridization, respectively, this phase exhibits nematic orbitals that allow $\sigma$ bonding with a sixfold coordination for the middle atoms in the $\mathrm{MoS}_{2}$ structure. With bond lengths longer than for silicene, the three nematic orbitals exhibit $\sigma$ band dispersions similar to those of LB silicene, which represents a common feature of $\sigma$ bonding in two-dimensional Si phases. On the other hand, the modified $p_{z}$ orbitals, or super $p_{z}$ orbitals, are prominently different from those of low-buckled silicene. Per Si atom, the $\mathrm{MoS}_{2}-\mathrm{Si}$ phase is lower in total energy than the low-buckled silicene, making it the most stable two-dimensional $\mathrm{Si}$ allotrope predicted so far. Our study demonstrates that $\mathrm{Si}$ atoms are capable of forming diverse types of $\sigma$ bonds even under ambient pressure conditions that are by themselves quite different from those formed by their smaller and larger cousins carbon and germanium. With yet unimagined bonding configurations on the playing field, we anticipate a lively discussion about the physics of such novel $\mathrm{Si}$ and Ge nanostructures as well as new ideas for the interpretation of structural and electronic properties of experimentally realized "epitaxial silicenes," and in particular on "multilayer silicenes" reported recently [29].

Importantly, the presence of an extended $\pi$ electronic system different from those in silicene and graphene will lead to properties that must still be explored. In a wider context, this finding not only opens opportunities in the engineering of novel nanostructures to be employed in future applications but also leads to intriguing fundamental questions related to the physics and chemistry of Si systems in general.

Note added. Recently, the growth of two-dimensional germanium layers on $\mathrm{Pt}(111)$ [30] and $\mathrm{Au}(111)$ [31] has been reported.

\section{ACKNOWLEDGMENTS}

We thank the anonymous referee for a comment with regard to the hypervalency of Si atoms. This work has been supported by the Japan Society for the Promotion of Science (JSPS) KAKENHI Grant No. 25.03351; the Strategic Programs for Innovative Research, the Ministry of Education, Culture,Sports, Science, and Technology (MEXT), Japan; and the Funding Program for Next Generation World-Leading Researchers Grant No. GR046. This work was also supported by the Computational Materials Science Initiative (CMSI), and Materials Design through Computics, MEXT, Japan. F.G. gratefully acknowledges a JSPS postdoctoral fellowship. The calculations have been performed using the Cray XC30 machine at the Japan Advanced Institute of Science and Technology.
[1] A. K. Geim and K. S. Novoselov, Nat. Mater. 6, 183 (2007).

[2] S. Cahangirov, M. Topsakal, E. Akturk, H. Sahin, and S. Ciraci, Phys. Rev. Lett. 102, 236804 (2009).

[3] A. Fleurence, R. Friedlein, T. Ozaki, H. Kawai, Y. Wang, and Y. Yamada-Takamura, Phys. Rev. Lett. 108, 245501 (2012).

[4] K. Takeda and K. Shiraishi, Phys. Rev. B 50, 14916 (1994).

[5] G. G. Guzman-Verri and L. C. Lew Yan Voon, Phys. Rev. B 76, 075131 (2007).

[6] P. Wetzel, S. Saintenoy, C. Pirri, D. Bolmont, and G. Gewinner, Phys. Rev. B 50, 10886 (1994).

[7] P. Vogt, P. De Padova, C. Quaresima, J. Avila, E. Frantzeskakis, M. C. Asensio, A. Resta, B. Ealet, and G. Le Lay, Phys. Rev. Lett. 108, 155501 (2012).

[8] C. L. Lin, R. Arafune, K. Kawahara, N. Tsukahara, E. Minamitani, Y. Kim, N. Takagi, and M. Kawai, Appl. Phys. Express 5, 045802 (2012).

[9] H. Jamgotchian, Y. Colignon, N. Hamzaoui, B. Ealet, J. Y. Hoarau, B. Aufray, and J. P. Bibeérian, J. Phys.: Condens. Matter 24, 172001 (2012).
[10] L. Meng, Y. Wang, L. Zhang, S. Du, R. Wu, L. Li, Y. Zhang, G. Li, H. Zhou, W. A. Hofer, and H.-G. Gao, Nano Lett. 13, 685 (2013).

[11] L. Chen, C. C. Liu, B. Feng, X. He, P. Cheng, Z. Ding, S. Meng, Y. Yao, and K. Wu, Phys. Rev. Lett. 109, 056804 (2012).

[12] L. Chen, H. Li, B. Feng, Z. Ding, J. Qiu, P. Cheng, K. Wu, and S. Meng, Phys. Rev. Lett. 110, 085504 (2013).

[13] C. C. Lee, A. Fleurence, R. Friedlein, Y. Yamada-Takamura, and T. Ozaki, Phys. Rev. B 88, 165404 (2013).

[14] D. Kaltsas and L. Tsetseris, Phys. Chem. Chem. Phys. 15, 9710 (2013).

[15] V. Ongun Özçelik and S. Ciraci, J. Phys. Chem. C 117, 26305 (2013).

[16] R. R. Holmes, Chem. Rev. 96, 927 (1996).

[17] B. Radisavljevic, A. Radenovic, J. Brivio, V. Giacometti, and A. Kis, Nature Nano. 6, 147 (2011).

[18] T.-M. Chuang, M. P. Allan, J. Lee, Y. Xie, N. Ni, S. L. Bud'ko, G. S. Boebinger, P. C. Canfield, and J. C. Davis, Science 327, 181 (2010). 
[19] J. P. Perdew, K. Burke, and M. Ernzerhof, Phys. Rev. Lett. 77, 3865 (1996).

[20] T. Ozaki, Phys. Rev. B 67, 155108 (2003).

[21] T. Ozaki et al., http://www.openmx-square.org/.

[22] See Supplemental Material at http://link.aps.org/supplemental/ 10.1103/PhysRevB.90.165423 for details on the convergence of the phonon calculations as a function of the size of the supercell.

[23] H. Weng, T. Ozaki, and K. Terakura, Phys. Rev. B 79, 235118 (2009).

[24] P. Blaha, K. Schwarz, G. K. H. Madsen, D. Kvasnicka, and J. Luits, WIEN2K, An Augmented Plane Wave+Local Orbitals Program for Calculating Crystal Properties, K. Schwarz, Vienna, 2001.
[25] M. Houssa, G. Pourtois, V. V. Afanas'ev, and A. Stesmans, Appl. Phys. Lett. 96, 082111 (2010).

[26] Y. Wang and Y. Ding, Solid State Commun. 155, 6 (2013).

[27] N. Marzari and D. Vanderbilt, Phys. Rev. B 56, 12847 (1997).

[28] A. Mujica, A. Rubio, A. Munoz, and R. J. Needs, Rev. Mod. Phys. 75, 863 (2003).

[29] P. De Padova, O. Kubo, B. Olivieri, C. Quaresima, T. Nakayama, M. Aono, and G. Le Lay, Nano Lett. 12, 5500 (2012).

[30] L. Li, S.-z. Lu, J. Pan, Z. Qin, Y.-q. Wang, Y. Wang, G.-y. Cao, S. Du, and H.-J. Gao, Adv. Mater. 26, 4820 (2014).

[31] M. E. Davila, L. Xian, S. Cahangirov, A. Rubio, and G. Le Lay, New J. Phys. 16, 095002 (2014). 